\title{
Efficient hybrid topology and shape optimization combining implicit and explicit design representations
}

Nguyen, Tuan T.; Bærentzen, J. Andreas; Sigmund, Ole; Aage, Niels

Published in:

Structural and Multidisciplinary Optimization

Link to article, DOI:

$10.1007 / \mathrm{s} 00158-020-02658-5$

Publication date:

2020

Document Version

Peer reviewed version

Link back to DTU Orbit

Citation (APA):

Nguyen, T. T., Bærentzen, J. A., Sigmund, O., \& Aage, N. (2020). Efficient hybrid topology and shape optimization combining implicit and explicit design representations. Structural and Multidisciplinary Optimization, 62, 1061-1069. https://doi.org/10.1007/s00158-020-02658-5

\section{General rights}

Copyright and moral rights for the publications made accessible in the public portal are retained by the authors and/or other copyright owners and it is a condition of accessing publications that users recognise and abide by the legal requirements associated with these rights.

- Users may download and print one copy of any publication from the public portal for the purpose of private study or research.

- You may not further distribute the material or use it for any profit-making activity or commercial gain

- You may freely distribute the URL identifying the publication in the public portal 


\title{
Efficient hybrid topology and shape optimization combining implicit and explicit design representations
}

\author{
Tuan T. Nguyen · J. Andreas Bærentzen · Ole Sigmund · Niels Aage
}

Received: date / Accepted: date

\begin{abstract}
This paper presents an interactive hybrid topology optimization method that, 1) employs density for topology optimization and, 2) in a seamless fashion uses a deformable simplicial complex for shape optimization. Omitting hole insertions during the shape optimization allows us to utilize adaptive mesh coarsening, which reduces the mesh size with up to seven times. The result is a combined method which can reduce computation time up to ten times in comparison to pure Lagrangian methods, while still producing adaptive meshes of good quality for analysis and design. Given the robustness of the method, we are able to perform topology optimization by explicit meshing and shape optimization on a mobile device at frame rates that allow for real-time user interaction. The resulting "Topopt Shape" app is available in the App Store for iOS devices.
\end{abstract}

\section{Introduction and related works}

An important aspect of structural optimization is how the material boundaries are represented. In general, two categories exist: implicit and explicit. Implicit representations make topology changes trivial, therefore they are largely adopted in topology optimization. Typical methods in this category are density methods, e.g. [7, 23], and certain fixed grid level set methods, e.g. [5, 22, 26]. On the other hand, explicit representations require complicated meshing schemes to deal with topology changes. This

Tuan T. Nguyen* . J. Andreas Bærentzen

Department of Applied Mathematics and Computer Science

Technical University of Denmark

Lyngby, Denmark

*E-mail: tntr@dtu.dk

Niels Aage · Ole Sigmund

Department of Mechanical Engineering,

Technical University of Denmark

Lyngby, Denmark problem is closely related to the interface tracking topic in computer graphics, where deformable meshes that support split and merge operations have been studied intensively $[13,19,27]$, since such methods allow the majority of the mesh to remain unchanged as the interface moves.

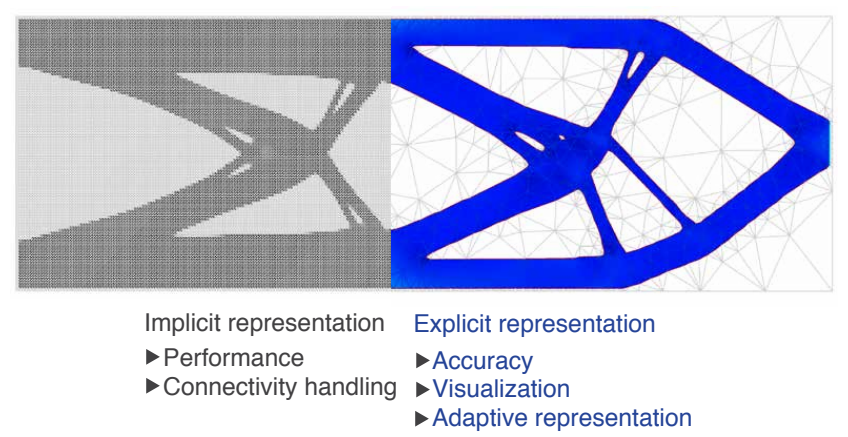

Fig. 1 Structure representations, implicit and explicit, with each their advantages

Despite the difficulty in handling topology changes, explicit representations are still desired for their well defined interfaces and the ability to perform accurate finite element analysis. Early approaches [4, 16, 28, 29] utilize an underlying level set function to resolve topology change, and a new explicit interface is generated in each iteration. Other approaches try to combine shape and topology optimization by iteratively introducing holes and then optimizing the shape without mesh change $[8,15,17]$. For these approaches, remeshing schemes are needed to ensure the quality of the mesh. Some recent proposals $[11,12,18]$ utilize Deformable Simplicial Complex (DSC), a deformable mesh method [19], to handle the mesh adaptation. As the DSC method offers mesh split and merge operations, this method can also change the topology, i.e close holes, while maintaining a good quality mesh throughout the optimization process. 
Though the DSC-based optimization provides promising results, one clear disadvantage of combining topology and shape optimization with explicit uniform meshing is its low computational performance [18]. In order to introduce holes, these methods need to maintain fine resolution meshes that neglect the advantage in adaptive representation of explicit meshes.

This paper presents a hybrid approach that utilizes the density representation for topology optimization and subsequently second order triangular mesh for shape optimization. The method consists of two steps. The first step is adopted from [3] for interactive topology optimization. However, the output is used to initialize the subsequent step while not necessarily converged. In the second step, we follow [12] for shape optimization, which includes shape changes and holes closing on a triangle mesh. The major difference of our method is that we do not require a fine mesh for shape optimization. Instead, we propose an adaptive resolution triangle mesh scheme that reduces the mesh size up to seven times compared to a pure Lagrangian method. This results in a better numerical performance, which can be up to ten time faster. Our contributions include

- A method to interactively bridge density optimization and shape optimization with deformable mesh

- An adaptive mesh updating scheme

Given the improvement of the performance, we are able to run $2 \mathrm{D}$ structural optimization with explicit mesh on mobile devices in real time with full interactivity during the topology optimization. We have published the Topopt Shape app for iOS devices at [2], and an Android app is planned.

\section{Overview of our method}

\subsection{Compliance optimization}

Given a configuration including domain layout, loads, supports, and passive material; our goal is to find the optimal structure represented by a triangle mesh that minimizes the structural compliance subject to a volume constraint, i.e.

$$
\underset{\mathbf{x}}{\operatorname{argmin}} \phi(\mathbf{x})=\mathbf{u}^{T} \mathbf{K}(\mathbf{x}) \mathbf{u}
$$

subject to : $\frac{V(\mathbf{x})}{V_{0}} \leq f$

$$
\begin{array}{ll}
: & \mathbf{K}(\mathbf{x}) \mathbf{u}=\mathbf{F} \\
: & \mathbf{x} \in\left[\mathbf{x}_{\min }, \mathbf{x}_{\max }\right]
\end{array}
$$

Here $\mathbf{x}$ contains the design variables which can be either density or shape variables; $\mathbf{K}$ is the global stiffness matrix; and $\mathbf{u}$ is the displacement found by solving the linear elastic plane stress equilibrium equation $\mathbf{K u}=\mathbf{F}$, where $\mathbf{F}$ is the load vector.

\subsection{General algorithm}

Fig. 2 shows our general algorithm. We first perform topology optimization with a fixed grid using a density method [23]. This process stops when the residual error is smaller than a threshold, which is not necessarily small, or whenever the user decides to switch to shape optimization.

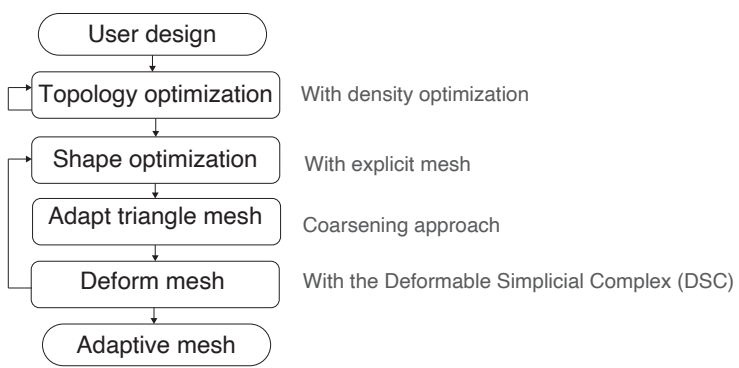

Fig. 2 General algorithm of proposed hybrid method

We estimate the topology using the density method, which represents the structure on a fixed grid, and the design variable $\mathbf{x}$ contains the material density of the elements. To allow for full interactivity during this phase, we adopt the approach from [3] without any modification. However, to boost performance, our implementation is in $\mathrm{C}++$ with the Suite-Sparse library [9] using the direct sparse Cholesky solver.

The density grid will be used as initialization for the shape optimization step (Sec. 3), which outputs the optimized structure represented by a triangle mesh. For this second step, we follow [12] and utilize the gradient-based optimization algorithm MMA [24] to compute updated positions of the interface vertices. The mesh is then deformed using the Deformable Simplicial Complex (DSC) method [19]. This shape optimization process also allows topology change and hole closing.

By neglecting holes insertion, the mesh does not need to be regularly dense (maintaining small triangles inside the domain). Instead, we propose an adaptive meshing scheme to achieve compact representations of the meshes (Sec. 4). The scheme is based on a mesh coarsening approach, which starts from a dense mesh and iteratively collapses edges, triggered by geometric criteria, c.f. section 4 .

\section{Shape optimization}

\subsection{Representation and mesh handling}

We represent the structure using piecewise linear polynomials, and based on this geometry discretization a triangulation is performed. As shape optimization needs to move the mesh, handling of mesh connectivity changes 
is required and poses a challenge. In the literature, there are several proposals for interface tracking [19], [27], [13], but here we employ the Deformable Simplicial Complex (DSC) method [19]. As opposed to other methods, DSC uses a triangular mesh to represent the modeling domain and requires minimal local change to ensure good quality of the mesh. Furthermore, it is publicly available on Github [6] as open source code.

In order to detect connectivity changes, the DSC maintains a triangle mesh on the whole domain and fixes the mesh locally if a vertex moves out of its 1-ring (all neighbor triangles) during mesh deformation. Each triangle is labeled to either solid or void material (Fig. 3). The DSC supports multiple materials, but in our method, we will utilize two materials only, namely solid and void. Given the new positions of interface vertices, the DSC will move the interfaces and automatically take care of the connectivity changes.

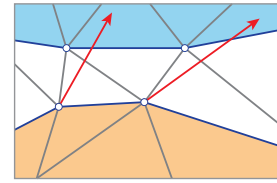

a) Vertex displacements b) move in 1-ring

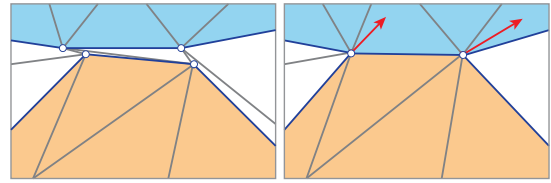

c) continue to move
Fig. 3 The DSC a) deforms the mesh; b) moves the vertices in their 1-ring and refines ill-shaped element; c) continues until the interfaces have moved to their destination. Image courtesy [21]

Fig. 3 illustrates the DSC algorithm. First, it moves the interface vertices as far as possible without creating inverted triangles. After resolving degenerated elements, DSC continues to move the mesh until it reaches the specified destination. DSC may requires several iterations to complete the deformation. This example demonstrates a simulation where two materials come in contact and shows how the DSC merges the interfaces.

For a detailed discussion of shape optimization with the DSC, the reader is referred to [12].

\subsection{Initialization from density grid}

The main novelty of this method is to allow for seamless switching between density based topology optimization and parameterized shape optimization. This requires an efficient and robust method to convert the density design into a shape representation. To transform the density result into a triangle mesh, we start with uniform mesh with edge length of 1 (the element size in density grid). Pixels with material density larger than 0.1 are used to label the triangle to solid material (density 1 denotes solid material and 0 means void material). Finally, we smooth the interface using the Taubin smoothing algorithm. Reference vertices corresponding to support, load, or passive material are simply snapped to the grid.

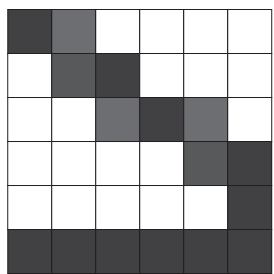

a) Density grid

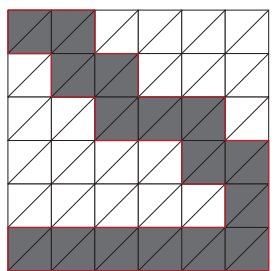

b) Uniform mesh

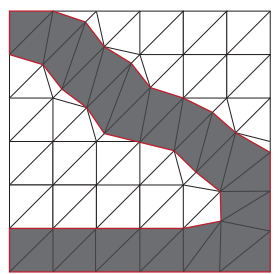

c) Smoothed interface
Fig. 4 Mesh initialization from a density grid

An alternative approach is mesh generation (with marching square and Delaunay triangulation, which may provide a better initial mesh. However, the implementation is overkill, and initialization is not essential in our method as the mesh will converge quickly to its optimized position anyway.

\section{Adaptive meshing scheme}

To achieve fast computation for the shape optimization, we employ an adaptive mesh strategy by a coarsening approach. That is, we start with a dense mesh and remove collapsible edges after each optimization iteration. We detect these edges by testing the mesh quality assuming the edge was removed. The coarsening routine is shown in Alg. 1

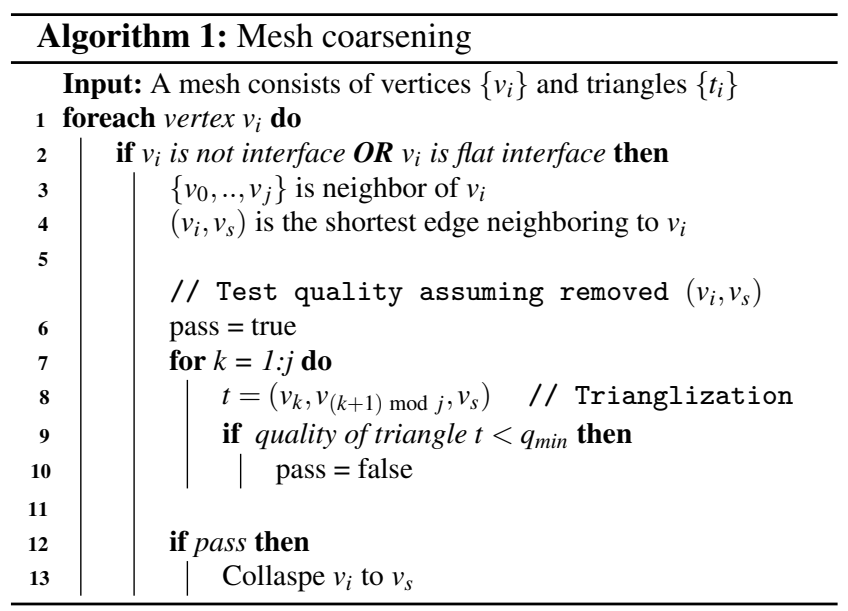

In our algorithm, we use triangle angles as the criteria for mesh quality. The angle threshold $q_{\min }$ can also be used to control the adaptivity of the mesh. From our experience, a minimum angle $q_{\min }=20^{\circ}$ results in a good balance between performance and finite element mesh quality. 


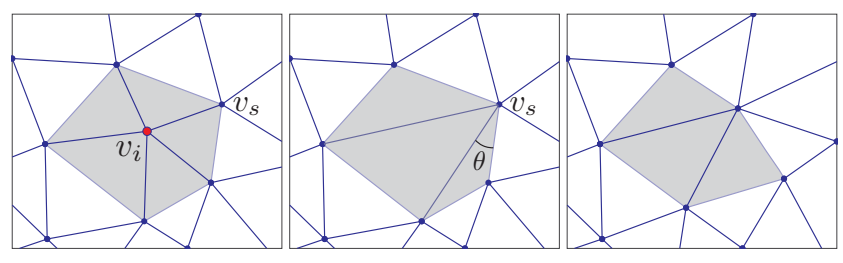

a) $v_{i}$ and neighbor

b) Triangulation

c) After smooth

Fig. 5 Edge collapse (gray area denotes the vertex 1-ring). a) Evaluating vertex $v_{i}$ and its neighbor. b) Triangulation of assumed collapse. c) After global smooth

The main ingredient in the adaptive scheme concerns the collapse of edges. Here, the shortest edge neighboring to a vertex will be considered for collapsing. Fig. 5 demonstrates the new triangulation if the edge is collapsed. We only perform the operation if the minimal angle $\theta<q_{\text {min }}$. After all vertices are evaluated, we perform a global mesh smoothing to improve the quality of the mesh. For interface vertices, we only collapse those with low curvature, i.e interface angle is larger than $175^{\circ}$.

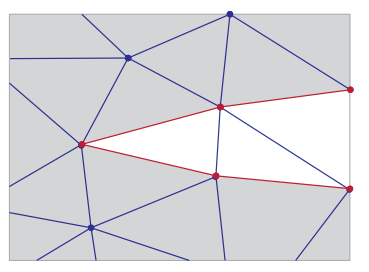

a) Interface triangle with degenerated corner

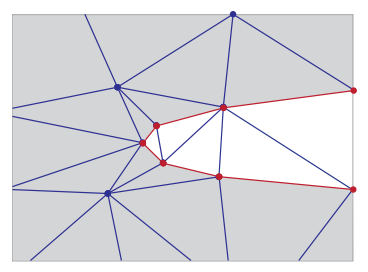

b) After needle alleviation
Fig. 6 Needle alleviation. The gray area denotes solid, and white is void material. Red edges denote interface

One issue of the coarsening approach is the appearance of sharp corners. To eliminate these bad features, we apply needle alleviation for interface vertices. Fig. 6 demonstrates the algorithm, where we split the two long edges of the degenerated triangle and then smooth the neighboring mesh. This procedure refines all vertices with interface angles less than $90^{\circ}$.

\section{Results and discussion}

We benchmark our method on three classical and well-studied examples: MBB beam, cantilever, and L-bracket with reasonable mesh sizes as we are aiming for interactive optimization. Fig. 7 shows the design problems, the outputs from density method, generated initial meshes, our hybrid method and a pure shape optimization from [12] (hereafter referred to as the explicit mesh method in this paper). All volume constraints are 0.5. All experiments are run on a single core $2.5 \mathrm{GHz} \mathrm{CPU}$ of an Apple iPad pro 11" (model A1980). We will discuss the results in term of accuracy, performance, and one property of our method: mesh adaptivity.

Table 1 Ratio of final compliance with respect to initial compliance (full domain). The smaller the better

\begin{tabular}{|c|c|c|c|c|}
\hline Method & Cantilever & L-bracket & MBB & $\begin{array}{c}\text { L-bracket } \\
\text { high res }\end{array}$ \\
\hline Density & 1.60 & 1.41 & 1.61 & 1.37 \\
\hline Explicit method & 1.55 & 1.37 & 1.47 & 1.43 \\
\hline Hybrid & 1.52 & 1.37 & 1.44 & 1.34 \\
\hline
\end{tabular}
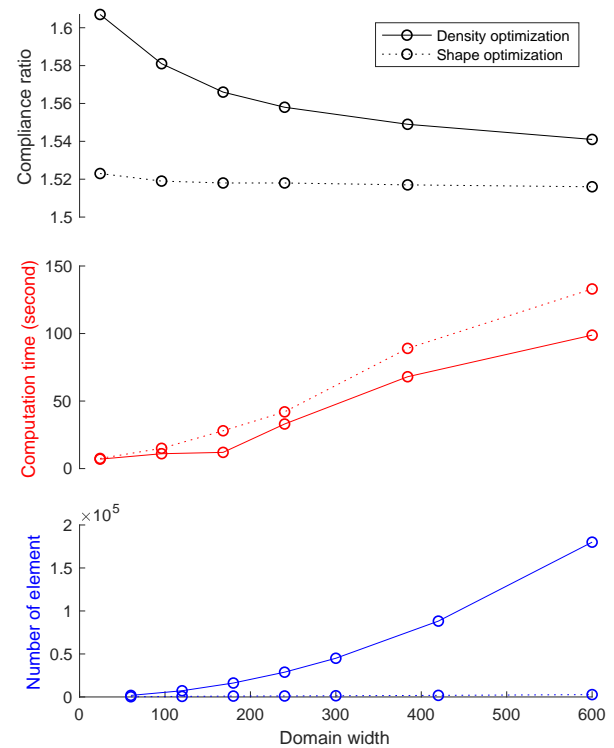

Fig. 8 Comparing density optimization (solid lines) and our shape optimization component (dotted lines) with different domain sizes

Accuracy: We use compliance as the criterion for comparison but not the absolute value due to the differences in discretization, filter and parameters between implementations. Instead, we compare the ratio of final compliance (with volume ratio $=0.5$ ) with respect to the full domain compliance (volume ratio $=1$ ) in Table 1 . We also perform experiments on the cantilever example with different choices of resolutions in Fig. 8.

The results represented in Table 1 show that the hybrid method achieves the best compliances, followed by the explicit mesh method, and that the density method comes last. Based on Table 1 and Figures 7 and 9, it is our interpretation that the explicit method is best when it comes to shape optimization whereas the density method is superior in terms of finding the topology. Our method takes advantage of both by using the topology from the density method followed by further shape optimization. Also, it should be noted that the reported values for the density approach are based on designs that have rather pronounced 

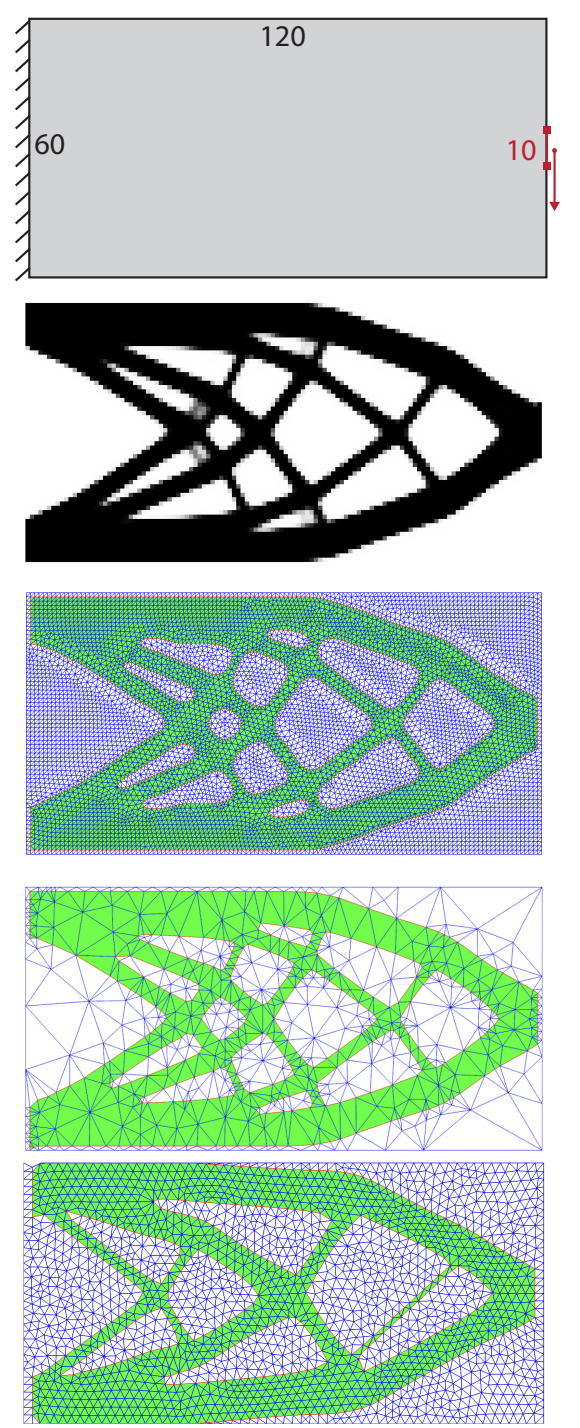

a) Cantilever
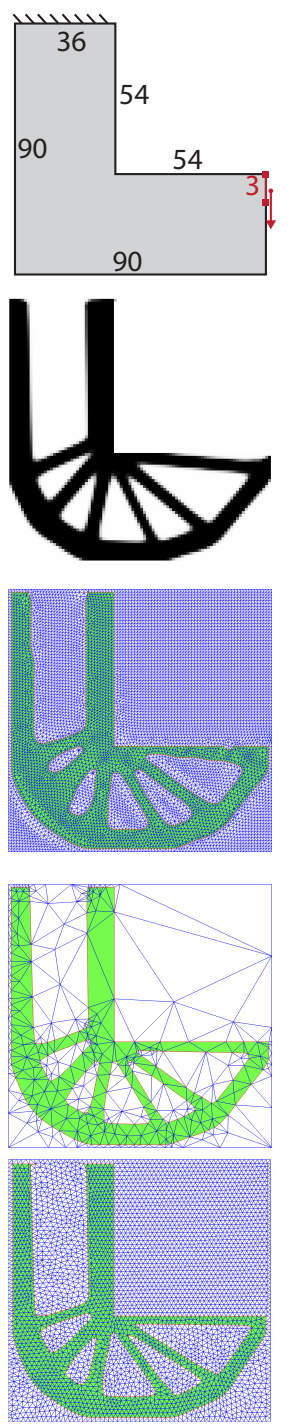

b) CLL
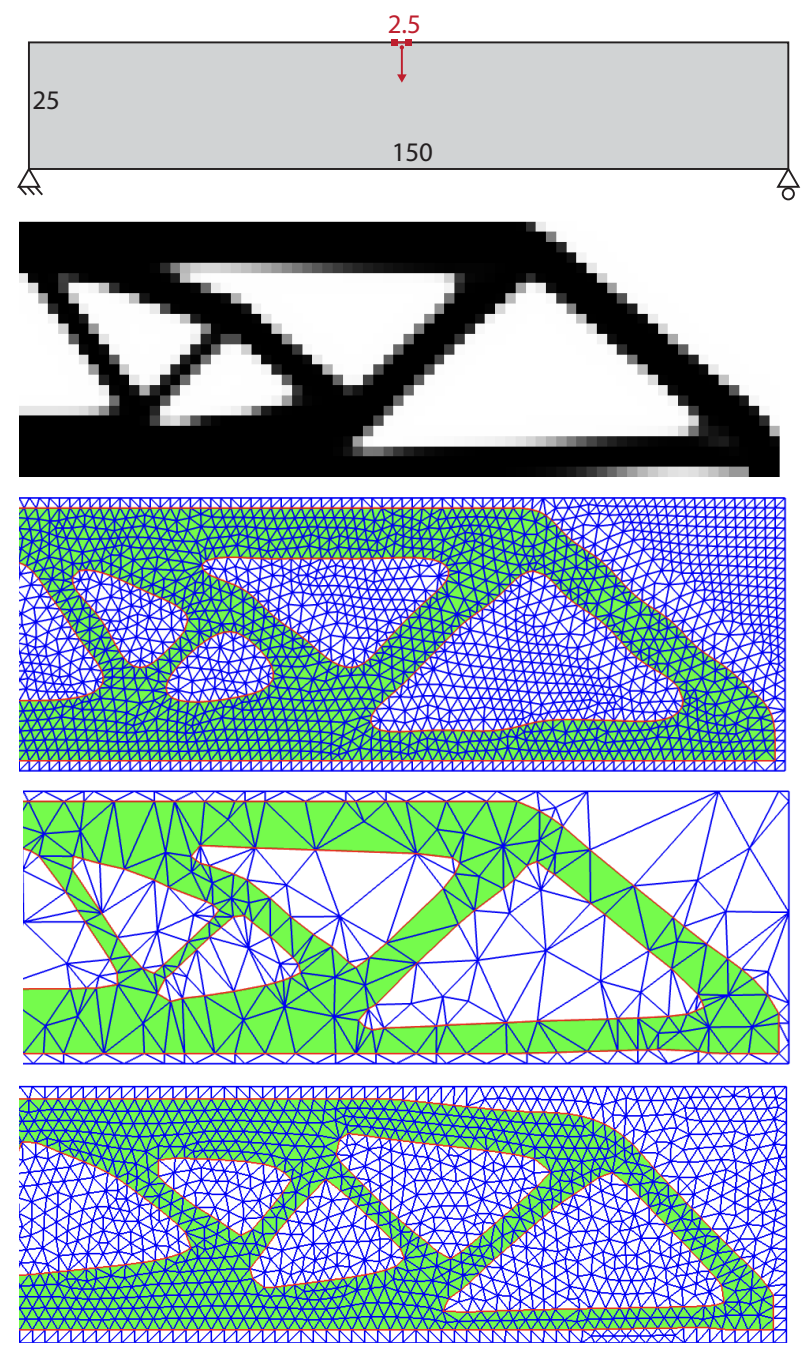

c) MBB (Only display half of the mesh)

Fig. 7 Comparison between our method and density method and the explicit mesh method. Row 1: Designs domain; row 2: Density output [23]; row 3: generated meshes from density outputs; row 4: Our method; row 5: Explicit mesh method [12]

regions of penalized grey-scale values that influence the obtainable compliance values.

Fig. 8 shows that with respect to resolution choices: our method performs better than density method, and computation times scale similarly. Besides, adaptive mesh helps reducing the number of elements significantly.

Note that the explicit mesh method is heavily parameter-dependent. Fig. 9 shows the optimizations of the L-bracket problem with the same design as Fig. 7b but with double resolution. The explicit mesh method produces a different structure (Fig. 9c) with even higher compliance than Fig. 7b (see Table. 1 last column). In short, the explicit mesh method requires fine-tuned parameters while the density and hybrid methods are less sensitive to the parameter choice.

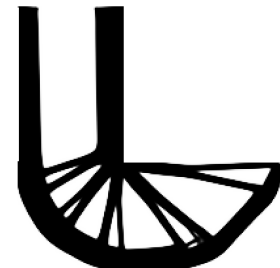

a) Density

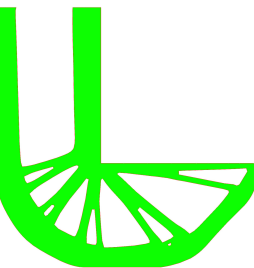

b) Hybrid method

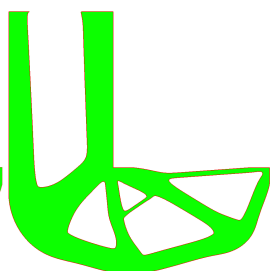

c) Explicit mesh
Fig. 9 L-bracket with doubled resolution compared to Fig. 7b

Performance: Table 2 shows computation time comparisons between the three methods, and the difference is significant. Although starting with four times higher mesh size, our method is around ten times faster than the explicit mesh method, and this number is scaled up with even larger problems. Importantly, it increases the frame 
rate to around $10 \mathrm{fps}$, which enables interactive topology optimization in real time. All implementations are in $\mathrm{C}++$ with the Suite Sparse solver, and the stopping criterion is visual assessment using the interactive Topopt Shape app.

Table 2 Performance comparison, total time [second] (total iterations). Third row represents only our second step, and density method is our first step

\begin{tabular}{|c|cc|cc|cc|cc|}
\hline Method & Cantilever & L-bracket & MBB & \multicolumn{2}{|c|}{$\begin{array}{c}\text { L-bracket } \\
\text { high res }\end{array}$} \\
\hline Density & 2.2 & $(110)$ & 2.3 & $(92)$ & 1.3 & $(185)$ & 9.8 & $(79)$ \\
\hline Explicit & 42.7 & $(33)$ & 52.2 & $(41)$ & 52.9 & $(38)$ & 793 & $(253)$ \\
\hline Hybrid & 5.3 & $(21)$ & 3.9 & $(40)$ & 4.0 & $(36)$ & 8.2 & $(38)$ \\
\hline
\end{tabular}

Compact output mesh: Omitting hole insertion in our second step enables mesh adaptivity. The explicit mesh method, in contrast, has to maintain a fine resolution mesh in order to insert holes by flipping specified triangles to void. Table 3 shows the comparison of mesh sizes, and the differences are significant. Even though our method starts with four times finer grid, the final mesh is three to seven times coarser than that of the explicit mesh method. This difference also scales with domain size (Fig. 8 last paragraph). A compact mesh not only reduces the memory usage, but also reduces the computation time for the linear elasticity, which is essential in topology optimization.

Table 3 Mesh size (Number of triangles [hundreds], solid / void )

\begin{tabular}{|l|c|c|c|c|c|}
\hline \multicolumn{2}{|l|}{ Method } & Cantilever & L-bracket & MBB & $\begin{array}{c}\text { L-bracket } \\
\text { high res }\end{array}$ \\
\hline Explicit & init & $37 / 3.7$ & $49 / 3.3$ & $47 / 5.6$ & $140 / 90$ \\
& final & $19 / 18$ & $25 / 53$ & $23 / 26$ & $68 / 150$ \\
\hline Hybrid & init & $74 / 76$ & $54 / 114$ & $40 / 41$ & $210 / 450$ \\
& final & $6.3 / 6.4$ & $3.7 / 3.6$ & $4.7 / 4.4$ & $7.6 / 7.2$ \\
\hline
\end{tabular}

Mesh convergence: Adaptive meshes generally contain more ill-shaped and larger elements than uniform meshes, hence they decrease the quality of the finite element solution. We analyze this issue with a mesh convergence study, where we examine the compliance error with respect to the average element size $h$ of the three structures in Fig. 7 rows 4 and 5. We utilize the recursive longest edge bisection algorithm [20] to refine the meshes without moving any vertex. With each refinement, $h$ is approximately halved.

Fig. 10 illustrates the Von Mises stress distribution of our design meshes and their finest discretizations, and Fig. 11 shows the relative compliance error with respect to average element size. The results show that the adaptive meshes converge similarly to uniform meshes. The MBB example does not follow this expectancy due to the point applied load and supports. The graph also shows that our design meshes

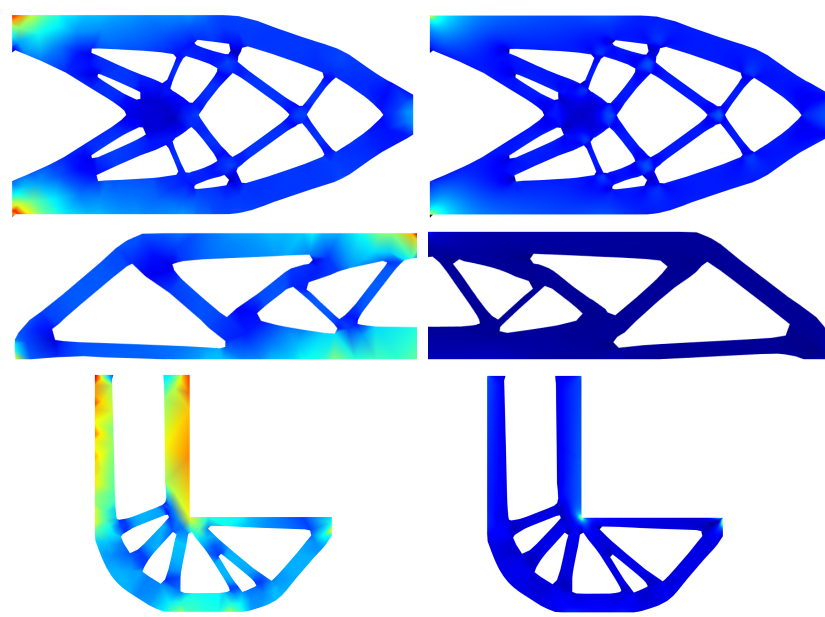

a) Design meshes

b) Finest discretizations

Fig. 10 Von Mises stress distribution of our design meshes (thousands of degrees of freedom) and the finest discretizations (million of degrees of freedom)

generally have slightly higher relative error than those of the explicit method.

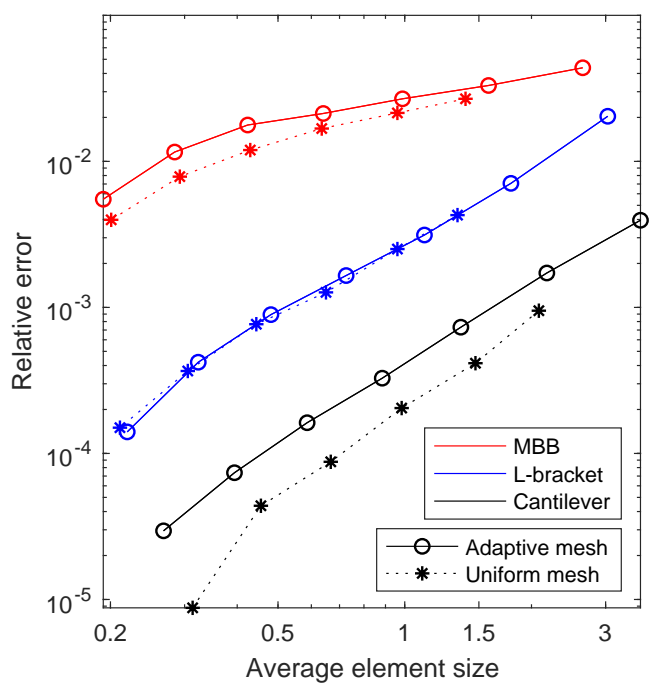

Fig. 11 Convergence plot of relative compliance error, log-log scale. Uniform meshes refer to [12]

Premature switching between density and shape: One of the key advantages of a fully interactive optimization strategy is that it allows the user to intervene at any time during the overall design process. For example, the user does not have to wait until the density solution has converged, but can at any time during the density process switch to shape optimization. Of course, if stopped too soon, this is likely to lead to poor local minima, c.f. the designs in Fig 12 where the compliance ratios (with respect to full volume compliance) are $1.55,1.53,1.52$ (top to bottom). However, 


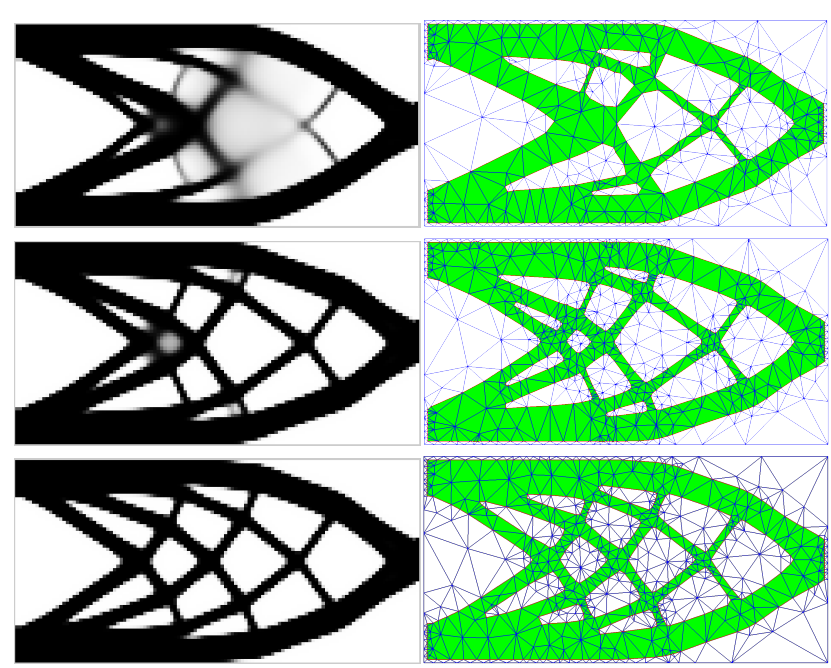

Fig. 12 Premature switching between density and shape. From the top, density optimization stops at 50,125, and 1500 iterations. The left column shows the final density grids, and the right column shows the output design meshes

such a trade-off between optimality and design control is likely to be a desired asset for many practitioners such as industrial designers and architects, for whom, reaching the best possible local minima might not be as essential as being able to influence the process as it happens.

\section{The Topopt Shape app}

In recent years, the high-end processors that power premium tablets and smartphones have surpassed the CPUs in most laptops and even some desktop processors. As a special example, Apple's A12X CPU, used in the iPad pro 2018, is faster than $92 \%$ of laptop processors. This fact makes mobile devices attractive, even for tasks like structural optimization, especially because mobile devices are so widely adopted.

From 1999 to 2015, the DTU TopOpt group [1] has maintained a web-based topology optimization app based on a client-server architecture [25], where all heavy tasks were performed on a server and the app simply showed the final result. In 2015, we published an interactive topology optimization application, the TopOpt and TopOpt3D app [3], for hand-held devices. These apps solves 2D and 3D compliance minimization problems using a density representation on a fixed grid, and the surface is generated using the marching cube algorithm. To our knowledge, TopOpt and TopOpt3D are the only apps for structural optimization on mobile devices. Since they were launched, these apps have been downloaded more than 15000 times.

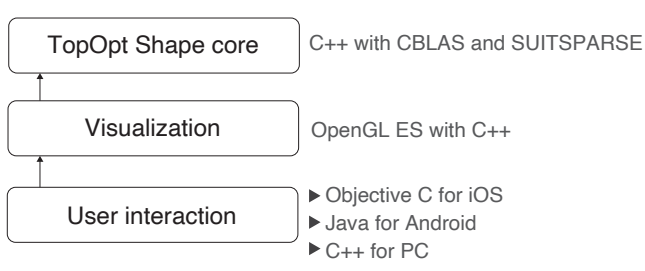

Fig. 14 TopOpt Shape app components

Given the obtained speed and the mesh representation, we are able to produce the new TopOpt Shape app. Users can iteratively update the design to achieve a desired output (Fig. 13). The results can be saved and shared. For implementation (14), the core component, which performs structural optimization, is written in $\mathrm{C}++$ leveraging high performance matrix libraries CBLAS and LAPACK; and the sparse solver CHOLMOD [10], which is part of he SuiteSparse library [14]. The visualization component is written in $\mathrm{C}++$ using OpenGL ES, which works on both Android and iOS. Other user interface components are written in Objective-C.

We have published the Topopt Shape app, demonstrated in Fig. 13, for iOS devices at https://apps.apple.com/dk/app/topopt-shape/id1445661360, and an Android app is planned.

\section{Conclusion}

This paper proposes a hybrid method that combines Eulerian and Lagrangian methods for structural optimization. We employ density optimization to estimate the topology and use this result as initialization for shape optimization with a triangle mesh. The second step restricts topology changes to hole closing and merging only. The challenge of mesh connectivity change handling is overcome by utilizing the Deformable Simplicial Complex, an explicit interface tracking method.

By omitting hole insertion during shape optimization, our approach enables mesh adaptivity that reduces the mesh size up to seven times. The scheme is based on a coarsening approach, and the adaptivity level can be controlled.

Our experiments show that density optimization provides more optimized topologies than explicit methods, but the compliance is higher due to pixelated representation and grey scale. The hybrid method combines the topology from density optimization and the shape representation from the explicit method, hence provides result with lower compliance.

In terms of performance, our method is up to ten times faster than pure triangle-based methods and makes interactive optimization possible in real time. With this ability, we have published an app named Topopt Shape for mobile devices in iOS AppStore. 


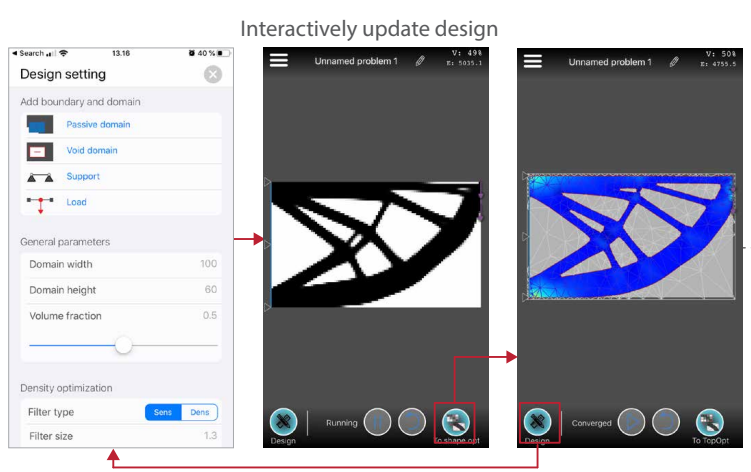

a) Build and update design

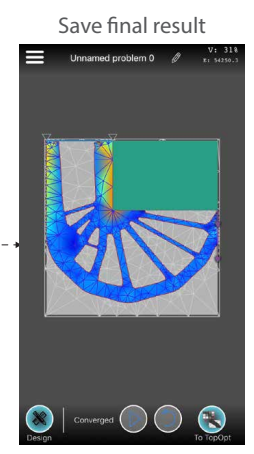

b) Desired result can be saved

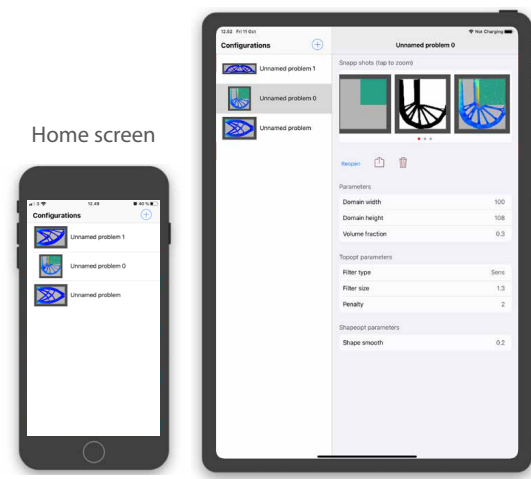

c) Home screen with saved configurations

Fig. 13 TopOpt Shape app demonstration

Acknowledgements The authors acknowledge the financial support from the Villum Foundation (InnoTop VILLUM investigator project).

Conflict of interest The authors declare that they have no conflict of interest.

Replication of results Section 6 introduces the TopOpt Shape app that uses the proposed method for structural optimization.

\section{References}

1. DTU topopt group website. http://www.topopt.mek.dtu.dk

2. Topopt shape app in ios appstore. https://apps.apple.com/dk/app/topopt- shape/id1445661360

3. Aage, N., Nobel-Jørgensen, M., Andreasen, C.S., Sigmund, O.: Interactive topology optimization on hand-held devices. Structural and Multidisciplinary Optimization (2013)

4. Allaire, G., Dapogny, C., Frey, P.: Topology and geometry optimization of elastic structures by exact deformation of simplicial mesh. Comptes Rendus Mathematique 349(17-18), 999-1003 (2011)

5. Allaire, G., Jouve, F., Toader, A.M.: Structural optimization using sensitivity analysis and a level-set method. Journal of computational physics 194(1), 363-393 (2004)

6. Bærentzen, J.A.: Deformable Simplicial Complex. https://github.com/janba/2D-DSC

7. Bendsoe, M.P.: Structural Optimization Optimal shape design as a material distribution problem. Structural Optimization 1, 193-202 (1989)

8. Bletzinger, K.U., Maute, K.: Towards generalized shape and topology optimization. Engineering Optimization 29(1-4), 201-216 (1997)

9. Chen, Y., Davis, T.A., Hager, W.W., Rajamanickam, S.: Algorithm 887: CHOLMOD, supernodal sparse cholesky factorization and update/downdate. ACM Transactions on Mathematical Software 35(3), 1-14 (2008)

10. Chen, Y., Davis, T.A., Hager, W.W., Rajamanickam, S.: Algorithm 887: Cholmod, supernodal sparse cholesky factorization and update/downdate. ACM Transactions on Mathematical Software (TOMS) 35(3), 22 (2008)

11. Christiansen, A.N., Bærentzen, J.A., Nobel-Jørgensen, M., Aage, N., Sigmund, O.: Combined shape and topology optimization of 3D structures. Computers \& Graphics 46, 25-35 (2015)

12. Christiansen, A.N., Nobel-Jørgensen, M., Aage, N., Sigmund, O., Bærentzen, J.A.: Topology optimization using an explicit interface representation. Structural and Multidisciplinary Optimization 49(3), 387-399 (2013)

13. Da, F., Batty, C., Grinspun, E.: Multimaterial mesh-based surface tracking. ACM Transactions on Graphics 33(4), 1-11 (2014)
14. Davis, T.A.: Suite sparse. http://faculty.cse.tamu.edu/davis/suitesparse.html

15. Eschenauer, H.A., Kobelev, V.V., Schumacher, A.: Bubble method for topology and shape optimization of structures. Structural Optimization 8(1), 42-51 (1994)

16. Ha, S.H., Cho, S.: Level set based topological shape optimization of geometrically nonlinear structures using unstructured mesh. Computers and Structures 86(13-14), 1447-1455 (2008)

17. Kim, D.H., Lee, S.B., Kwank, B.M., Kim, H.G., Lowther, D.A.: Smooth boundary topology optimization for electrostatic problems through the combination of shape and topological design sensitivities. IEEE Transactions on Magnetics 44(6), 1002-1005 (2008)

18. Lian, H., Christiansen, A.N., Tortorelli, D.A., Sigmund, O., Aage, N.: Combined shape and topology optimization for minimization of maximal von Mises stress. Structural and Multidisciplinary Optimization 55(5), 1541-1557 (2017)

19. Misztal, M.K., Bærentzen, J.A.: Topology-adaptive interface tracking using the deformable simplicial complex. ACM Transactions on Graphics 31(3), 1-12 (2012)

20. Nambiar, R.V., Valera, R.S., Lawrence, K.L., Morgan, R.B., Amil, D.: An algorithm for adaptive refinement of triangular element meshes. International Journal for Numerical Methods in Engineering 36(3), 499-509 (1993)

21. Nguyen, T.T., Dahl, V.A., Bærentzen, J.A., Trinderup, C.H.: Multi-phase Volume Segmentation with Tetrahedral Mesh. In: BMVC, pp. 2-3 (2018)

22. Osher, S., Fedkiw, R.: Level set methods and dynamic implicit surfaces, vol. 153. Springer Science \& Business Media (2006)

23. Sigmund, O.: Topology optimization-theory, methods, and applications (2003)

24. Svanberg, K.: The method of moving asymptotes - a new method for structural optimization. International journal for numerical methods in engineering 24(2), 359-373 (1987)

25. Tcherniak, D., Sigmund, O.: A web-based topology optimization program. Structural and Multidisciplinary Optimization 22 (2001)

26. Wang, M.Y., Wang, X., Guo, D.: A level set method for structural topology optimization. Computer methods in applied mechanics and engineering 192(1-2), 227-246 (2003)

27. Wojtan, C., Thürey, N., Gross, M., Turk, G.: Deforming meshes that split and merge. ACM Transactions on Graphics 28(3), 1 (2009)

28. Xia, Q., Shi, T., Liu, S., Wang, M.Y.: A level set solution to the stress-based structural shape and topology optimization. Computers and Structures 90-91(1), 55-64 (2012)

29. Yamasaki, S., Nomura, T., Kawamoto, A., Sato, K., Nishiwaki, S.: A level set-based topology optimization method targeting metallic waveguide design problems. International Journal for Numerical Methods in Engineering 87(9), 844-868 (2011) 